\title{
Image Formation Based on Atomic Resolution Core-loss Electron Energy Loss Spectroscopy
}

\author{
M.P. Oxley,* K. van Benthem,* M. Varela,* S.D. Findlay,,* L.J. Allen** and S.J. Pennycook* \\ * Condensed Matter Sciences Division, Oak Ridge National Laboratory, PO Box 2008, Oak Ridge, \\ TN 37831 \\ ** School of Physics, The University of Melbourne, Victoria, 3010, Australia
}

Aberration correction has allowed the formation of scanning transmission microscope (STEM) probes of an Ångström or less, leading to the prospect of core-loss electron energy loss spectroscopy (EELS) measurements at true atomic spatial resolution. The interpretation of images based on EELS however is complicated by many factors including nonlocality of the effective inelastic potential [1], channeling of the incident electrons and inelastic scattering of the probe to high angles [2]. In addition, size of the core state from which ionization occurs becomes crucial in the image formation process for small probes [3].

In Fig. 1 we show images formed by the integrated EELS signal from the $\mathrm{O} K$-shell and La $\mathrm{N}_{45}$ edges in [100] zone axis oriented $\mathrm{LaMnO}_{3}$. The experimental data was taken using VG Microscopes' HB501UX fitted with a Nion aberration corrector, with a probe size of approximately $1.3 \AA$. The line scans were taken in the $<011>$ direction across alternating La and $\mathrm{Mn} / \mathrm{O}$ columns using a Gatan Enfina with a collection semi-angle of 12 mrad. We see that despite the large difference in binding energies for these two edges ( $\sim 500 \mathrm{eV}$ for $\mathrm{O} K \mathrm{vs} \sim 100 \mathrm{eV}$ for $\left.\mathrm{La} \mathrm{N}_{45}\right)$, the contrast and width of the images are very similar. We also see that there is a reduction in intensity above the columns containing the atoms from which the signal has originated. Such "volcanoes" have been seen in simulations based on inelastic STEM images of isolated atoms [3,4], but the formation of these features in the bulk has not been previously reported.

In Fig. 2 we show the simulated single atom images formed for the same probe and detector parameters used in the experiment. This allows an examination of the image features due to the ionization interaction rather than the dynamical scattering of the probe by the sample. The solid background indicates the projected electron density of the bound state orbital from which the ionization has occurred. We see that the width of the $\mathrm{O} K$-shell orbital is far smaller than that of the La $\mathrm{N}_{45}$ orbital as expected from the relative binding energies. However the $\mathrm{O} K$-shell image has a half width at half maximum that is significantly broader than that of the La $\mathrm{N}_{45}$ image. This is counter to simple impact parameter models which predict increasing image localization for larger binding energies [5]. In addition we see that there is a distinct reduction in the intensity of the $\mathrm{O} K$ shell signal directly above the atomic site. There is however no corresponding dip in the La $\mathrm{N}_{45}$ signal above the atomic location. The dip in the La $\mathrm{N}_{45}$ image above the La column seen in Fig. 1 can not be due to the nature of the interaction alone.

We will discuss the mechanisms leading to the formation of these images and the need for simulation in order to correctly understand images based on core-loss spectroscopy at atomic resolution. Using $\mathrm{LaMnO}_{3}$ as a model we will show that images formed from different edges from the same atomic species can lead to totally different interpretations of the sample's structure. 
References

[1] M.P. Oxley et al., Phys. Rev. Lett. 94: (2005) 203906 (4 pages).

[2] S.D. Findlay et al., Ultramicroscopy 104, (2005) 126.

[3] E.C. Cosgriff et al., Ultramicroscopy 102, (2005) 317.

[4] H. Kohl and H. Rose, Adv. Imaging and Electron Phys., 65 (1985) 173.

[5] R.F. Egerton, Electron Energy Loss in the Electron Microscope. Plenum Press. New York 1986

[6] This research work was sponsored by the U.S. DOE Basic Energy Sciences, under contract

DE-AC05-00OR22725 with Oak Ridge National Laboratory, managed and operated by UT-Battelle, LLC. L.J.A. acknowledges the support of the Australian Research Council.
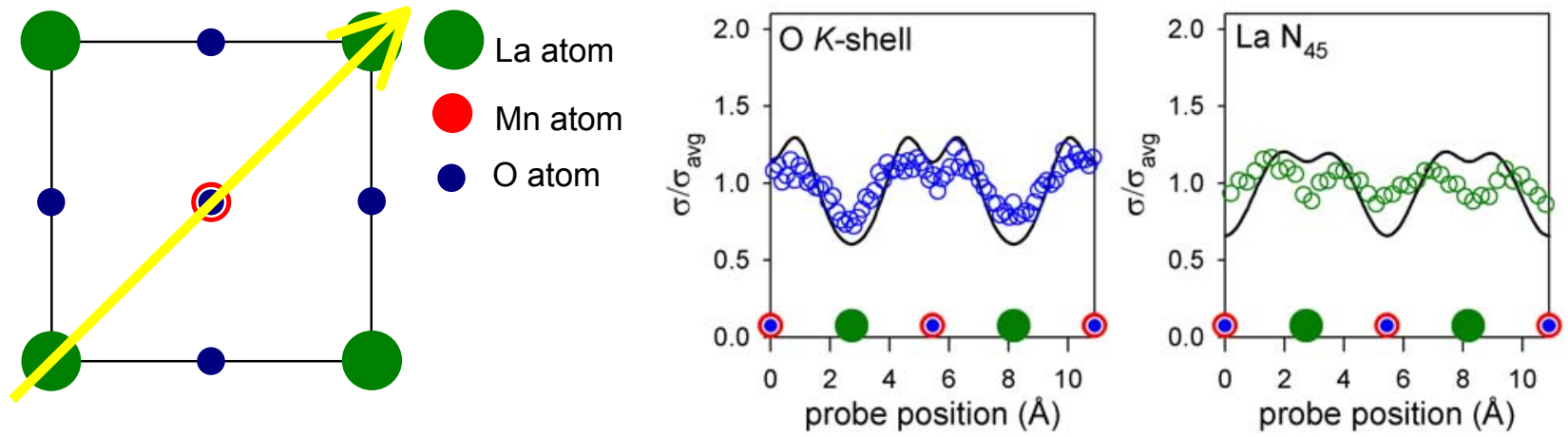

FIG. 1. Images formed from integrated core-loss EELS line scans in the $<011>$ direction for $\mathrm{LaMnO}_{3}$ in the [100] zone axis orientation. The scan was taken using VG Microscopes' HB501UX operating at $100 \mathrm{keV}$ with a probe size of approximately $1.3 \AA$. Reductions in intensities above the atomic columns containing the atomic species of interest can be seen in both cases.
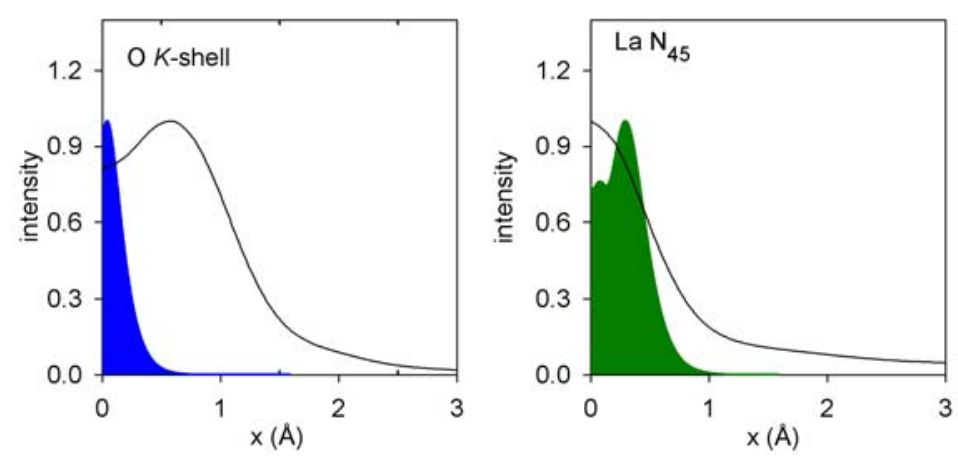

FIG. 2. Simulated integrated STEM EELS images for isolated oxygen and lanthanum atoms. The projected electron density of the orbitals from which the ionization has occurred is indicated by the solid background. 